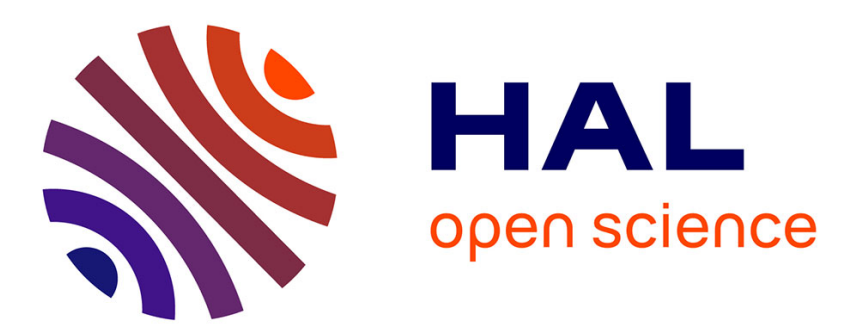

\title{
Les Russes du Caucase du Sud: du malheur d'avoir un empire (et de le perdre)
}

\author{
Silvia Serrano
}

\section{To cite this version:}

Silvia Serrano. Les Russes du Caucase du Sud: du malheur d'avoir un empire (et de le perdre). Revue d'Etudes Comparatives Est-Ouest, 2008, 39, pp.121 - 147. 10.4074/S033805990800106X . hal01533719

\section{HAL Id: hal-01533719 https://hal.science/hal-01533719}

Submitted on 6 Jun 2017

HAL is a multi-disciplinary open access archive for the deposit and dissemination of scientific research documents, whether they are published or not. The documents may come from teaching and research institutions in France or abroad, or from public or private research centers.
L'archive ouverte pluridisciplinaire HAL, est destinée au dépôt et à la diffusion de documents scientifiques de niveau recherche, publiés ou non, émanant des établissements d'enseignement et de recherche français ou étrangers, des laboratoires publics ou privés. 


\title{
Les Russes du Caucase du Sud : DU MALHEUR D'AVOIR UN EMPIRE (ET DE LE PERDRE)
}

\author{
SILVIA SERRANO
}

Maître de conférences en science politique, Université d'Auvergne-ClermontFerrand 1, et chercheur au CERCEC-EHESS (serrano.sil@gmail.com)

\begin{abstract}
Résumé : Les populations russes du Caucase méridional, vieillissantes, appauvries et numériquement affaiblies, rencontrent de grandes difficultés pour s'adapter à la situation née de l'éclatement de l'URSS. Aujourd'hui citoyens des nouveaux États indépendants, les Russes jouissent à ce titre de droits similaires aux populations locales mais, auparavant massivement employés dans l'armée ou l'industrie lourde, ils sont frappés de plein fouet par les transformations économiques et géopolitiques. Le recul de la russophonie, qu'il provienne ou non de politiques délibérées de promotion de la culture nationale, dont témoigne par exemple la disparition progressive des " écoles russes », les marginalise un peu plus. À la différence des autres minorités nationales, ces anciennes élites de l'empire sont en outre dépourvues des ressources symboliques et politiques fournies par l'ethnicité pour négocier leur place dans les nouvelles sociétés.
\end{abstract}


Selon le dernier recensement soviétique de 1989,341 200 Russes vivaient en Géorgie (soit 6,3 \% de la population totale) et constituaient le troisième groupe après les Arméniens ; ils formaient le deuxième groupe national en Azerbaïdjan, avec 392000 personnes (5,6 \% de la population, dont 295500 à Bakou). En Arménie, la république la plus ethniquement homogène, on ne dénombrait que 51600 Russes (1,6 \%). Or ces populations, installées pour certaines depuis le début du $\mathrm{XIX}^{\mathrm{e}}$ siècle, se sont réduites comme peau de chagrin depuis l'effondrement de l'URSS : en 2001, les Russes n'étaient plus que 14660 en Arménie, 141700 en Azerbaïdjan, et, en 2002, seuls 67671 résidaient toujours en Géorgie où ils avaient perdu $80 \%$ de leur effectif (voir tableaux en annexe).

Les débordements nationalistes de la fin des années 1980 puis les conflits ont provoqué l'émigration massive d'une population craignant pour sa sécurité. En Azerbaïdjan par exemple, les départs ont lieu dès les pogroms anti-arméniens et la répression par l'armée soviétique du « janvier noir » ${ }^{1}$ de 1990 : entre 1989 et 1995, 169000 Russes, 15000 Ukrainiens et 3000 Biélorusses quittent le pays. L'effondrement des économies a continué à pousser hors des nouveaux États tous ceux qui le pouvaient, y compris les représentants des nationalités titulaires.

Au-delà des raisons conjoncturelles, la marginalisation sociale et professionnelle des Russes dans les nouveaux États indépendants a encouragé leur exode. Le départ des populations originaires de la métropole accompagne fréquemment les processus de décolonisation mais le phénomène a ici un caractère particulier qui renvoie à la spécificité de la construction impériale russe et soviétique, à la place qu'y occupaient les Russes et à la situation originale du Caucase dans l'empire.

Après 1991, les « pieds rouges $»^{2}$ russes ne parviennent plus à concevoir leur avenir dans les républiques du Caucase. L'adaptation à la nouvelle donne s'est avérée ardue pour toutes les minorités mais plus encore pour ces héritiers d'un empire discrédité, souvent encore persuadés d'en avoir été l'avant-garde. Leur situation sociale s'est brusquement dégradée et ils ne sont pas parvenus à retrouver leur place dans des régions où ils étaient chez eux ; ils étaient d'autant plus affaiblis que la Russie ne les a guère soutenus.

1. Dès février 1988, plusieurs dizaines d'Arméniens sont massacrés à Soumgaït, puis dans d'autres villes du pays, parmi lesquelles Bakou en décembre 1989. Ce n'est toutefois qu'après les pogroms contre les Arméniens, et alors que le mouvement national échappe à tout contrôle, que l'armée soviétique intervient en janvier 1990, faisant des centaines de victimes, principalement azéries.

2. Par analogie avec les « pieds noirs », l'expression a été utilisée pour désigner les populations russes installées dans les territoires des États issus de l'URSS, devenus indépendants en 1991. 


\section{SERVITEURS DE L'EMPIRE, ÉLITE DU PROLÉTARIAT, LUMPENPROLÉTARIAT : HISTOIRE D'UN DÉCLASSEMENT}

La promotion de certaines élites indigènes, par le biais de la cooptation ou de l'assimilation, n'est pas la moindre des caractéristiques de l'empire russe puis soviétique. Cette politique a des répercussions importantes sur le statut des populations issues de la métropole.

\subsection{LES PREMIERS PEUPLEMENTS :}

POPULATIONS RURALES «COMMUNAUTAIRES » ET ÉLITES MÉTISSÉES

Les premiers peuplements massifs de Russes au Caucase du Sud datent de l'intégration de la région dans l'Empire tsariste entre 1801 (intégration du royaume de Kartlie-Kakhétie) et 1828 (traité de Turkmentchaï).L'administration tsariste cherche alors à peupler ces nouvelles marches de populations russophones et chrétiennes supposées plus loyales. Après la paix d'Adrinople (1829), au terme d'échanges de populations, les chrétiens (Grecs, Arméniens, sectateurs russes $^{3}$ ) remplacent les musulmans qui se réfugient dans l'Empire ottoman (Mouradian, 1990, pp.19-20). C'est dans ce contexte que plusieurs milliers de sectateurs - Doukhobores et Molokanes en particulier - quittent les régions de Tambov, de Saratov ou de Voronej pour s'installer en Transcaucasie ; leurs déplacements, volontaires ou coercitifs, sont organisés par le décret du 20 octobre 1830 (Skakov, 2003)4. Quelque soixante-dix ans plus tard, les réformes de Stolypine entraînent une seconde vague de migration de populations rurales vers l'Azerbaïdjan. Plus d'une centaine de villages russes orthodoxes sont créés au début du $\mathrm{XX}^{\mathrm{e}}$ siècle en Azerbaïdjan où se concentre l'essentiel de la population rurale russe de Transcaucasie.

Un deuxième groupe est constitué par les militaires. Après l'intégration du royaume de Kartlie-Kakhétie en 1801 suivie par celle de la principauté d'Imérétie (1814), des garnisons sont établies à l'ouest de la Géorgie et des régiments sont stationnés à l'est, à Signakhi, Manglissi et Tiflis ${ }^{5}$. Après la répression qui succède à la rébellion du régiment Semenovskij (1820) puis des Décembristes (1825), des officiers et soldats sont envoyés au Caucase méridional. Malgré une mortalité élevée - notamment dans les régions marécageuses de l'ouest où sévissaient la malaria et la dysenterie - et en dépit des rotations, un certain nombre de soldats avait vocation à rester sur place, en particulier les Cosaques qui servaient en Transcaucasie. À cet effet, un décret de 1838 prévoyait la création de «bourgs militaires » destinés à abriter les familles des soldats.

3. Il s'agit d'adeptes de sectes dissidentes de l'Église orthodoxe apparues au XVIII siècle au sud de la Russie et dont l'origine est incertaine. Elles rejettent la liturgie et l'Église, prônent le pacifisme et ont une organisation économique et sociale communautaire.

4. En Géorgie, des villages doukhobores ou molokanes sont fondés en Djavakhétie, en Kakhétie et aux alentours de Tiflis. En Azerbaïdjan, les villages molokanes se situent au nord-ouest du pays et, en Arménie, au nord, vers le Lori et autour du lac Sévan.

5. Tbilissi, le nom géorgien, deviendra officiel en 1936. 
L'intégration dans l'Empire entraîne également l'arrivée de fonctionnaires russes puis, peu à peu, d'une «petite intelligentsia » qui rassemble des télégraphistes ou des techniciens. Au sein de la communauté russe, les militaires servant au Caucase forment l'élite et comptent dans leurs rangs des écrivains, tels Pouchkine ou Lermontov - officier des dragons -, qui ont contribué dans leurs écrits à façonner une mythologie romantique caucasienne (Layton, 1994). La présence de nombreux aristocrates et intellectuels a un impact sur l'image et les représentations du Caucase. Celui-ci, perçu comme une contrée certes exotique, n'en fait pas moins partie intégrante de l'Empire si bien que la géographie culturelle russe demeure intimement liée au Caucase. Aujourd'hui encore, y renoncer n'a pas la même valeur « symbolique » que le renoncement à l'Asie centrale. Les élites indigènes, du moins chrétiennes, sont assimilées à celles de l'Empire ${ }^{6}$, entrâ̂nant un grand métissage culturel. Les nobles arméniens - le comte LorisMellikov - et géorgiens - le général Ivan Andronnikov (Andronikachvili), les princes Tsitsianov (Tsitsichvili) ou Tchavtchavadzé - participent intensivement aux campagnes militaires russes, y compris pour certains, tel le prince Grigol Orbéliani, à la conquête du Caucase du Nord.

Le rapprochement des élites amène donc, dès 1820-1830, une partie des Géorgiens et des Arméniens à se russifier, à l'instar des autres nationalités qu'ils côtoient - Tchèques, Allemands ou encore Polonais. Quant aux Russes, certains s'enorgueillissent d'épouser des Géorgiennes :A. Griboïedov prend ainsi pour femme la fille du poète $\mathrm{A}$. Tchavtchavadzé. Tiflis, véritable capitale de la vice-royauté sous le prince Vorontsov (1844-1854), qui devient alors une ville européenne et un centre de la culture russe, est l'illustration de ce brassage. En somme, en Transcaucasie, les Russes ont pu se sentir non seulement en territoire conquis, mais bien chez eux, dans un espace politique et culturel qu'ils partageaient avec des populations locales parfois largement assimilées et dont l'altérité semblait appelée à disparaître, ce qui rend peu pertinent le cadre d'identification ethnique.

\subsection{LA PÉRIODE SOVIÉTIQUE : PRÉSENCE MILITAIRE ET INDUSTRIALISATION}

L'ère soviétique connaîtra d'autres vagues de russification. Après la Révolution, les premières sont liées aux famines de 1927-1930 en Ukraine, avec des flux provenant principalement du Kouban puis, au début des années 1930, du sud de l'Oural. D'autres déplacements de population ont lieu durant la Seconde Guerre mondiale dans le cadre des mesures d'évacuation du gouvernement soviétique.

Les politiques d'industrialisation de l'après-guerre s'accompagnent d'une arrivée massive d'ingénieurs et d'ouvriers russes travaillant dans les grandes unités de production telle l'usine d'aviation de Tbilissi (évacuée de

6. 30000 nobles géorgiens voient leurs titres de noblesse reconnus en 1859 .

Volume 39, Mars 2008 
Taganrog), dans les mines de Kvartchéli (aujourd'hui en territoire abkhaze) et dans les nouvelles villes industrielles (Roustavi en Géorgie, Soumgaït en Azerbaïdjan, Leninakan en Arménie, etc.). Dès l'après-guerre, la grande majorité des Russes de Transcaucasie constitue une population urbaine, souvent rassemblée dans les quartiers périphériques construits autour des usines. Enfin, une part non négligeable d'entre eux est composée de militaires dans les multiples bases déployées dans cette région frontalière de l'OTAN et, notamment, à Tbilissi où se trouve le siège du District militaire de Transcaucasie. Par ailleurs, la loi prescrit que le service militaire, d'une durée de deux ans (trois ans jusqu'en 1964), doit être effectué en dehors de sa république d'origine, de sorte que nombre de conscrits arméniens, azéris ou russes sont, par exemple, envoyés en Géorgie. Les bases militaires sont entourées de toute une série d'infrastructures (école, marchés, etc.) qui leur sont destinées ainsi qu'aux populations russes. Autrement dit, ces dernières occupent deux «niches professionnelles » inhérentes à la construction de l'empire russe, puis soviétique : l'armée et l'industrie.

\subsection{LA MARGINALISATION DUE À LA MONTÉE EN PUISSANCE}

DES ÉLITES RÉPUBLICAINES ET À LA PERTE DE PRESTIGE DE L'IDÉOLOGIE SOVIÉTIQUE

Après la Seconde Guerre mondiale, les populations russes sont confrontées aux politiques d'indigénisation et à la montée en puissance des élites nationales. La tendance démographique est au renforcement des peuples titulaires en raison, d'une part, de leur taux de natalité élevé en Arménie et en Azerbaïdjan et, d'autre part, de la migration des nationalités du Caucase méridional vers leur république éponyme ; le reflux des populations non caucasiennes, y compris des Russes, favorise ce mouvement (Anderson \& Silver, 1996). De surcroît, l'exode rural ne s'accompagne pas, au Caucase méridional, d'une arrivée massive de Slaves comme cela a pu être le cas ailleurs.

La proportion des Russes baisse depuis 1959 en Géorgie et en Azerbaïdjan (respectivement de 8,9 \% et de $17,5 \%$ entre 1979 et 1989) et se réduit de façon encore plus visible en Arménie où elle chute de 25,7 \% entre 1979 et 1989 (Anderson \& Silver, 1996). À Tbilissi, la population russe décline même en nombre absolu entre 1959 et 1970 (passant de 125700 à 124 300) (Suny, 1989). En Azerbaïdjan, la décroissance de la population russe, également imputable à une natalité plus faible que celle de la nationalité titulaire, devient massive dans les années 1970-1980 (Skako, 2003). Le reflux des Russes a donc débuté bien avant la perestroïka, anticipant ainsi, comme en Asie centrale, une évolution politique.

7. En 1959, $71 \%$ des Russes d'Arménie, $88 \%$ de ceux installés en Azerbaïdjan et $79 \%$ de ceux résidant en Géorgie vivent en ville. Ces chiffres atteignent respectivement $85 \%, 95 \%$ et $86 \%$ en 1989 (ANDERSON \& Silver, 1996). 
La position sociale des Russes est également fragilisée par les politiques de promotion de la nationalité titulaire. En Géorgie par exemple, en 1985, $79,1 \%$ des membres du Parti communiste sont géorgiens, ce pourcentage augmentant au fur et à mesure que l'on s'élève dans la hiérarchie. La prépondérance des Géorgiens est également notable dans l'enseignement supérieur où, en 1979, ils constituent 82,6 \% des étudiants et, en 1987, 94 \% de ceux de l'Université de Tbilissi (Jones, 1992). La création d'instituts de formation tel l'Institut du transport ferroviaire de Tbilissi (le TBIJDT qui deviendra ensuite le GPI, l'Institut polytechnique d'État), ouvert dès le milieu des années 1950, permet la formation de cadres locaux dans le secteur de l'industrie. Peu à peu, les Russes se trouvent exclus des fonctions dirigeantes, y compris dans des branches stratégiques comme l'extraction pétrolière en Azerbaïdjan. L'armée échappe dans une certaine mesure à ces changements, la grande majorité des échelons intermédiaires et supérieurs restant russes. Néanmoins, elle est affectée par un autre phénomène, le discrédit croissant du modèle soviétique.

Depuis les années 1970, en effet, celui-ci est concurrencé par d'autres modèles de réussite économique dans lesquels les Caucasiens excellent, en particulier l'entrepreneuriat individuel et la «débrouille». Ainsi les Russes, parce qu'ils ne peuvent rivaliser avec les «nouveaux riches » caucasiens, sont l'objet d'un certain mépris en raison de leur situation matérielle tandis que, pour les élites locales, la réussite économique et son étalage compensent la soumission coloniale. À la fin de l'époque soviétique, ni les ingénieurs ni les militaires n'occupent de positions enviables du point de vue économique, comme en témoigne cette histoire drôle :

« Dans une école géorgienne, la maîtresse demande aux écoliers ce que fait leur père :

- Mon père à moi, il est expert en marchandises, dit le premier.

- Le mien est directeur d'un entrepôt, dit un deuxième.

- Et moi, mon père, il est directeur de magasin, dit un troisième.

- Moi, mon père est ingénieur...

La classe éclate de rire.

- Les enfants, dit la maîtresse, ce n'est pas bien de rire du malheur des autres $»^{8}$.

Toutefois, pendant que les Caucasiens voient en eux les représentants d'un empire en déclin, les Russes continuent à se considérer comme l'élite du pays : s'il point dès les années 1970, leur déclassement n'est pas perçu comme tel avant 1991.

Jusqu'à l'effondrement de l'URSS, dans certains pays dont l'Azerbaïdjan, les plans de carrière de l'élite incluaient également les stratégies matrimoniales et il était de bon ton d'épouser une Russe (ou une Arménienne) pour s'élever socialement. Cependant, ce phénomène n'était pas dénué

8. Cité par Regamey, 2007.

Volume 39, Mars 2008 
de contradictions : si la gente masculine locale accueillait partout avec enthousiasme les cars de touristes russes, les bljadovozy $y^{9}$, pour l'ensemble de la population, un mariage avec une femme russe demeurait synonyme de mésalliance comme en témoigne le film L'Amour au premier regard de Rézo Esadzé (1975). Les mariages des femmes caucasiennes avec des Russes étaient encore plus rares et mal acceptés par la société.

\subsection{UnE SITUATION SOCIALE ET ÉCONOMIQUE PEU ENVIABLE}

Les deux principaux secteurs d'activité des populations russes ont été frappés de plein fouet après 1991. Avec la dislocation de l'armée soviétique et la constitution des armées nationales, les militaires russes sont progressivement contraints de quitter le Caucase du Sud. La fermeture des bases russes provoque ainsi le départ de plus de 70000 personnes d'Azerbaïdjan (Galstyan, 2004). Un nombre non négligeable de militaires de carrière reste pourtant sur place, généralement parce qu'ils sont mariés à des Caucasiennes et ont des attaches familiales. Ils se retrouvent de la sorte dans une position délicate dans la mesure où ils participent à la constitution des nouvelles armées nationales, voire combattent dans leurs rangs (c'est le cas des militaires russes qui servent sous le drapeau géorgien en Abkhazie) tout en étant soupçonnés d'un manque de loyauté envers leur nouvel État.

La production industrielle s'est totalement interrompue dès le début des années 1990, les usines fermant les unes après les autres ${ }^{10}$. La situation des Russes devient particulièrement vulnérable du fait de la nature de leurs activités professionnelles antérieures, mais aussi de la précarité de leurs réseaux de solidarité. À la différence des autres populations, ils n'ont généralement pas de famille à la campagne qui pourrait partiellement compenser l'absence d'économie monétarisée et certaines études soulignent leur paupérisation grandissante. En Arménie, à la fin des années 1990, 60 à $70 \%$ des familles russes sont estimées pauvres et $20 \%$ ont des revenus inférieurs au seuil de pauvreté (Galstyan, 2004). Alors que tous ceux, à commencer par les jeunes, qui entrevoient des perspectives en Russie émigrent massivement depuis plusieurs décennies, la population russe du Caucase méridional vieillit et est majoritairement féminine. En Azerbaïdjan, $63 \%$ de la population russe est féminine ; en Géorgie, cette part est encore plus forte puisqu'elle atteint $72 \%$, dont $60 \%$ sont âgés de plus de 40 ans (Sakartvelos mosaxleobis, 2003).

Dans les capitales, les Russes, qui habitent généralement des immeubles non entretenus et dont la valeur se déprécie, sont victimes de la différen-

9. Les « putocars ». C'est ainsi qu'étaient appelés les autocars transportant des femmes touristes. 10. Selon l'IDA (Association internationale de développement), le PIB a baissé de plus de $50 \%$ entre 1990 et 1993. Voir par exemple le rapport de l'IDA (Banque mondiale) de février 2007, «Arménie, récolter les fruits des réformes passées » (http://siteresources.worldbank.org/EXTIDAFRENCH/Resources/Armenia_fr.pdf). 
ciation des prix sur les marchés immobiliers du Caucase et de la Russie alors que l'appartement, bien souvent le seul bien possédé, tient lieu d'assurance. Au début des années 1990, les prix étaient à peu près équivalents partout, si bien que certains vendirent leur appartement pour en acheter un en Russie. Aujourd'hui, les écarts de prix empêchent de telles transactions, ce qui rend d'autant plus hypothétique le projet migratoire.

Parallèlement à la dégradation des conditions de vie sur place, les relations avec la Russie se distendent. Après l'effondrement de l'URSS, les pays du Caucase décident que tous les résidents peuvent, à moins d'y renoncer explicitement, obtenir la citoyenneté sans aucune condition particulière (examen de langue ou autre). En théorie, il est dans un premier temps possible d'adopter la citoyenneté de son choix mais la validité des passeports soviétiques ayant été prolongée pendant plusieurs années, les gens ne prennent pas immédiatement conscience des nouveaux enjeux qui n'apparaissent au grand jour qu'avec la fermeture progressive des frontières des pays nouvellement indépendants. Toujours est-il que les personnes de diverses nationalités qui ont acquis la citoyenneté de leur État de résidence jouissent, au regard de la loi, des mêmes droits que les populations titulaires, les trois pays du Caucase reconnaissant l'égalité des droits de leurs ressortissants indépendamment de leur origine. En revanche, aucun n'accepte la double citoyenneté, même si de nombreux individus ont réussi à obtenir plusieurs passeports.

Chaque crise des relations bilatérales a montré les limites de la citoyenneté unique, rendant par exemple plus complexe le maintien des liens avec les proches vivant au-delà des frontières. Paradoxalement, ceux-ci semblent s'étioler moins facilement lorsque la famille a émigré en Europe ou en Israël, plutôt qu'en Russie. Plusieurs facteurs sont responsables de cet éloignement : les habitudes épistolaires se sont perdues au moment de l'interruption des liaisons postales au début des années $1990 ;$ l'envol des prix des transports, le renforcement des frontières, la fermeture régulière des postes frontières et, dans le cas de la Géorgie, l'imposition de visas ont rendu aléatoires les déplacements. Le cas le plus extrême est celui de la Géorgie, les Russes de citoyenneté géorgienne souffrant, au même titre que les autres, de la rupture des relations avec la Fédération de Russie à l'automne $2006^{11}$.

11. Dans un climat déjà tendu, en rétorsion à l'expulsion, en septembre 2006, de quatre de ses ressortissants accusés d'être des agents du renseignement militaire, la Fédération de Russie rappelle son ambassadeur, décide d'instaurer un blocus économique et de suspendre toutes les liaisons ferroviaires, routières, aériennes, maritimes, postales et financières avec la Géorgie ; sous couvert de vérification de leur enregistrement, la Russie se livre sur son territoire à de vastes opérations de contrôle et d'expulsion de Géorgiens qui sont menées avec une grande brutalité et entraînent plusieurs décès. Voir FIDH, Migrations en Russie. Populations fragilisées, premières victimes des crises politiques externes et internes, mai 2007. La version française est consultable sur http://www.fidh.org/IMG/pdf/Migranrussie472fr2007.pdf/

Volume 39, Mars 2008 
Les possibilités de s'installer légalement en Russie sont réduites et se heurtent à de nombreux obstacles bureaucratiques (quotas pour l'obtention d'un permis de résidence ou de travail), tous ne bénéficiant pas du soutien d'une famille sur place susceptible de les aider à obtenir les documents nécessaires.

\section{L'INEXORABLE RECUL DE LA RUSSOPHONIE}

Leur méconnaissance de la langue nationale est l'un des principaux écueils sur lesquels se brise l'intégration des populations russes. À l'époque soviétique, leur langue maternelle - dont l'usage était certes limité par la mauvaise maîtrise qu'en avait une grande partie des populations autochtones - servait de langue véhiculaire ${ }^{12}$. Le recul démographique des populations russophones et la rupture des liens avec la Russie ont mené au déclin de la russophonie. Il est vrai que la place du russe varie fortement selon les pays. S'il reste la langue des élites en Azerbaïdjan, c'est de moins en moins le cas en Arménie où se font sentir les effets de l'éloignement géographique de la Russie et de l'arrivée d'Arméniens non russophones de la diaspora, y compris dans les instances du pouvoir ; quant à la Géorgie, dont les relations avec Moscou sont particulièrement conflictuelles, elle présente le cas extrême d'une éviction presque totale du russe de l'espace public. En dépit de ces différences, la tendance est partout au renforcement de la langue nationale, qu'il prenne la forme d'une évolution spontanée due à l'affaiblissement des attaches avec la Russie ou qu'il constitue un objectif délibéré des politiques de nationalisation, compliquant d'autant les conditions de vie de ceux qui ne la maitrisent pas ${ }^{13}$.

\subsection{UNE NOUVELLE LÉGISLATION EN MATIÈRE DE LANGUE NATIONALE}

La monopolisation du pouvoir par les élites des ethnies titulaires s'est accompagnée d'un déclin du russe. La question linguistique est particulièrement vive en Arménie et en Géorgie où la langue nationale est un marqueur fort de l'identité en raison, notamment, de son originalité radicale ${ }^{14}$. La préservation de la langue et d'un alphabet propre a joué un grand rôle dans le maintien de l'identité de la nation au point que, en 1978, lorsque les

12. Pour des données chiffrées concernant la maîtrise du russe à l'époque soviétique, voir ANDERSON \& Silver, 1996.

13. Selon certaines estimations, $15 \%$ de la population de la Géorgie ne connaîtraient pas assez bien le géorgien pour l'utiliser dans la vie courante. Voir Korth, STEPANIAN \& MusKHELISHVILI, 2005.

14. Au XIX ${ }^{\mathrm{e}}$ siècle, en Géorgie, les campagnes de russification se heurtent aux tentatives de promotion de la langue nationale, l'une des causes qu'embrassent les intellectuels nationalistes. L'écrivain Ilia Tchavtchavadzé définit l'identité nationale par la formule « une langue, une confession, une patrie », tandis que I. Goguébachvili diffuse son manuel Deda Ena (La langue maternelle). 
autorités soviétiques ont envisagé de modifier la législation dans le cadre de la politique de russification et de retirer de facto au géorgien et à l'arménien leur statut de langue nationale, des mobilisations importantes ont eu lieu dans les deux républiques. La langue russe est donc victime de cette forte idéologisation, même si les deux pays n'ont pas les mêmes enjeux. La situation est différente en Azerbaïdjan du fait, entre autres, d'une moindre différenciation linguistique et d'un investissement plus faible dans le discours national. Toutefois, avec la réforme de l'alphabet en 1991 qui a mené à l'abandon du cyrillique au profit de l'alphabet latin, la volonté de rompre avec l'espace culturel russe est patente ${ }^{15}$.

Dès l'indépendance, le législateur attribue au russe une place secondaire par rapport à la langue éponyme. En Géorgie où aucune loi n'est spécifiquement consacrée à la question linguistique, la Constitution de 1995 reconnaît le géorgien comme langue nationale, ainsi que l'abkhaze en Abkhazie. En Arménie, la loi en vigueur, qui date de 1993, est caractéristique de l'investissement de la question linguistique et de l'humeur nationaliste d'alors. Non seulement elle dispose que l'arménien est la langue nationale, mais elle interdit l'usage d'autres langues dans l'administration et même sur les panneaux de signalisation, les affiches publicitaires, les étiquetages, etc. ${ }^{16}$ Un service gouvernemental est spécifiquement chargé d'en contrôler l'application - citons, à titre d'exemple, la condamnation de la firme Swatch à payer une forte amende en 2006 pour n'avoir pas traduit sa marque en arménien ${ }^{17}$. En 1998, alors qu'il était candidat à la présidence, R. Kotcharian s'était engagé à faire du russe la seconde langue officielle mais le projet de loi élaboré en ce sens est resté lettre morte après son élection comme le relate, entre autres, la Nezavisimaja gazeta du 26 mai 2001. En 2002, en Azerbaïdjan, était adoptée une loi très offensive en faveur de l'azéri, manifestement motivée par la persistance relative du russe. Les populations russophones y ont vu une atteinte à leurs droits, notamment parce qu'elle interdisait la diffusion en d'autres langues d'émissions de télévision ou de radio (article 6$)^{18}$.

Toutes les législations adoptées rendent obligatoire l'usage de la langue d'État dans l'administration (articles 3 et 4 de la loi arménienne, article 1 de la loi azerbaïdjanaise, article 12 de la loi géorgienne sur le service public du 31 octobre 1997), malgré des dispositions qui visent la défense des langues minoritaires. En conséquence, la langue nationale s'impose pendant

15. La loi sur la langue officielle du 22 décembre 1992 accorde toutefois aux Azerbaïdjanais le droit de choisir leur langue d'enseignement.

16. Se reporter à http://www.parliament.am/hdoc/Laws/ru/pqbkl5.html

17. Pour les détails de cette affaire, voir http://www.advertology.ru/article27341.htm

18. Sous la pression du Conseil de l'Europe, semble-t-il, le Milli Medjlis (Parlement) a toutefois modifié la loi en 2003, autorisant $25 \%$ des émissions dans d'autres langues ; http://www. bezcenzury.info/pages/print.shtml?200511/p05111835.htm

Volume 39, Mars 2008 
que s'amenuise la tolérance à l'égard de l'usage du russe. En Géorgie par exemple, des arrangements locaux permettaient, sous E. Chevardnadzé, de rédiger en russe la correspondance administrative mais cette mesure a été remise en question par la nouvelle équipe de M. Saakachvili, porté au pouvoir en 2003. Ne pas maîtriser la langue nationale est un handicap de plus en plus pesant à mesure que l'ensemble des textes officiels et administratifs sont rédigés dans cette langue. Concrètement, quand bien même ce n'est pas une condition explicitement prévue par la loi, il devient impossible de travailler dans la fonction publique si l'on ne possède pas la langue officielle. Peu à peu, la réussite aux tests de langue est requise pour être recruté ou reconduit dans ses fonctions, ce qui a des conséquences particulièrement graves pour les Russes, souvent salariés de l'État.

\subsection{UNE RUSSOPHONIE EN DÉCLIN}

Le maintien ou non du russe dans la sphère publique ne dépend toutefois pas exclusivement des législations en vigueur, ni même de la présence effective de populations russes. L'exemple de la presse illustre les différentes tendances à l'œuvre dans les trois États. En Géorgie, la presse locale russophone datant de l'époque soviétique disparaît peu à peu, victime de la crise générale de la presse écrite. Ainsi, l'abonnement semestriel aux principaux périodiques russophones correspondait, en 2001, au salaire moyen, de sorte que seules les organisations pouvaient s'abonner. Les quotidiens ont vu fondre leur lectorat et leurs ressources s'amenuiser ${ }^{19}$. Leur périodicité s'est ralentie puisqu'ils ne sortaient plus que deux ou trois fois pas semaine; les tirages et la distribution ont, eux aussi, été réduits ${ }^{20}$. Ces journaux peinent à résister à la concurrence de la presse russe en provenance de Russie. Pour ce qui est des autres médias, certaines stations de radio locales diffusent encore des programmes en russe; il s'agit pour l'essentiel d'émissions musicales, celles consacrés à l'information ayant été supprimées. La radio publique diffuse dix minutes par jour en russe, une demi-heure le samedi. En outre, une chaîne russophone pro-gouvernementale, Alania TV, a commencé à émettre en 2005 à l'attention des Ossètes. Enfin, la télévision russe ne peut être captée qu'avec une antenne parabolique et en payant un abonnement, ce qui la rend inaccessible aux ménages modestes.

19. En 2001, les journalistes de Svobodnaja Gruzija, subventionné par l'État, et de Večernij Tbilissi, subventionné par la municipalité, n'avaient pas été payés depuis deux ans. Informations fournies par E. Adelkhanov.

20. La presse russophone n'est diffusée qu'à Tbilissi et, dans une moindre mesure, à Roustavi et Batoumi. D'autres journaux publiés en Géorgie ont une diffusion encore plus confidentielle : Kavkazskij Akcent ou Mnogonacional'naja Gruzija sont publiés par des ONG, Kazak Gruzii est le journal du Parti communiste, Zarja Vostoka est financé par l'ambassade russe et tiré à 1000 exemplaires de même qu'un digest de la presse géorgienne, tandis que Zakavkazskie Voennye Vedomosti, soutenu par le ministère de la Défense de la Fédération de Russie, a cessé de paraître en 2003. Les journaux de loisir, en revanche, conservent un certain lectorat. 
En revanche, en Azerbaïdjan, plusieurs dizaines de journaux et de revues sont publiés en russe, dont plus de douze dans la capitale (Vestnik, Sodružestvo, Zerkalo, Exo, etc.) ; des émissions quotidiennes de radio et de télévision sont destinées à un auditoire russophone et les principales châ̂nes russes (ORT, RTR, NTV) sont relayées sans restriction. Notons que les médias russophones ne s'adressent pas exclusivement, loin s'en faut, aux Russes. À ce titre, l'Azerbaïdjan, où se maintient une russophonie autochtone, présente des similitudes avec certains pays d'Asie centrale, qui ne sont pas sans rappeler les processus d'acculturation de l'époque soviétique.

La situation est plus contrastée en Arménie où une piètre connaissance du russe était inconcevable parmi les élites urbaines à l'époque soviétique. Néanmoins, les Arméniens ont été victimes de l'absence d'investissement de la Fédération de Russie dans la défense du russe. En dépit de la volonté proclamée par les autorités d'œuvrer à sa promotion ${ }^{21}$, rien n'a été concrètement entrepris, y compris quand la demande était forte. Ainsi les retards de paiement des droits des chaînes télévisées russes ont justifié l'arrêt de leur diffusion en Arménie comme en Géorgie. En 2001, la diffusion d'ORT a été limitée à Erevan et RTR était menacée en raison de son endettement (Nezavisimaja gazeta, 26 mai 2001).

Le recul du russe marginalise ses locuteurs. Témoin de cette régression, la télévision géorgienne diffuse désormais des feuilletons russes doublés en géorgien, les jeunes générations - de Géorgiens mais aussi d'autres minorités non russes - n'ayant même plus une maîtrise sommaire de cette langue. Ignoré, le russe a été remplacé par les autres langues véhiculaires que sont devenus le géorgien, l'arménien, voire le turc selon les régions. Or pour les populations ne comprenant pas le géorgien, trouver un appartement ou un emploi devient un tour de force et il n'est pas rare de voir, dans la rue, des Russes demander qu'on leur lise ce qu'ils pensent être une annonce d'emploi écrite en géorgien. Si la loi prévoit des interprètes dans les tribunaux, dans les faits, les inculpés sont souvent amenés à signer des documents qu'ils ne peuvent pas lire et les procès sont souvent reportés faute d'intercompréhension. On se fait remarquer à Tbilissi en parlant russe dans la rue. À Bakou, la population rurale a contribué à « azerbaïdjaniser » une ville auparavant russophone. Deux groupes coexistent dans deux mondes parallèles, les interactions étant réduites au minimum. Ainsi, les populations russophones se désintéressent généralement de la vie politique locale et sont beaucoup plus au fait de l'actualité de la Fédération de Russie dont elles ne détiennent pourtant pas la citoyenneté.

21. Depuis 2003, le soutien à la langue russe constitue un des objectifs prioritaires de Moscou. L'année 2007 a été décrétée année du russe par le président V. Poutine ; http://www. kremlin.ru/text/psmes/2006/12/116272.shtml

Volume 39, Mars 2008 


\subsection{LA DISPARITION DES ÉCOLES RUSSES}

La situation semble d'autant plus irréversible que les institutions qui auraient pu contribuer à la pérennisation du russe, en particulier au sein du système éducatif, sont en perte de vitesse. L'un des héritages soviétiques les plus prégnants est l'existence d' ' écoles nationales » où l'enseignement est prodigué dans les langues des différentes nationalités. Les plus nombreuses étaient les écoles russophones où la totalité du cursus s'effectuait en russe. Si à l'époque soviétique, le statut et la fonction des écoles russophones variait fortement d'une république à l'autre, soulignons qu'elles n'étaient pas fréquentées exclusivement par des élèves russes mais bien par l'ensemble des nationalités. En Arménie et en Azerbaïdjan, la mâ̂trise du russe était une condition sine qua non de toute ascension sociale et les écoles russes étaient recherchées pour leur niveau jugé supérieur à celui des autres écoles. En Géorgie, en revanche, une part non négligeable des élites nationales, animée par des sentiments nationalistes, préférait envoyer ses enfants à l'école géorgienne, la maîtrise de sa langue nationale étant considérée comme un signe de distinction sociale plus pertinent que celle du russe. La différence majeure ne tient donc pas seulement aux données statistiques sur la maîtrise du russe dans chaque république, mais aux implications sociales de celle-ci ${ }^{22}$.

Le principe d'un enseignement dans les langues nationales n'a pas nécessairement été directement remis en cause au début des années 1990 et ce, au nom du respect des cultures et des langues des minorités. Cependant, la montée en puissance de la langue d'État et les nouvelles exigences de promotion sociale l'ont rendu caduc. En Arménie, la loi sur la langue de 1993 impose l'arménien comme langue d'instruction, sauf pour les Russes, les enfants issus de couples mixtes ou les réfugiés, pour lesquels fonctionnent encore quelques écoles ${ }^{23}$. Les élèves arméniens continuent toutefois d'apprendre le russe, au même titre que les autres langues étrangères. En Azerbaïdjan, les écoles russophones ont longtemps enregistré de bonnes performances qui ne sont pas sans rapport avec la volonté des autorités d'adopter une nouvelle loi sur la langue. Ainsi, en 2001, 125000 écoliers dans 431 écoles (soit $13 \%$ des élèves azerbaïdjanais) fréquentaient encore les écoles russophones (Nezavisimaja gazeta, 26 mai 2001) et il existait des sections russes dans presque tous les établissements du supérieur ${ }^{24}$. On considérait généralement que les bacheliers sortant du système scolaire

22. Entre 30 et $50 \%$ des Arméniens, Géorgiens et Azerbaïdjanais disaient maîtriser le russe comme seconde langue en 1989. Voir ANDERSON \& SiLVER, 1996.

23. Des écoles proposant un apprentissage approfondi du russe ont ouvert leurs portes ( $16 \mathrm{en}$ 2001) ainsi que, à titre expérimental, des écoles bilingues qui ont essuyé de vives critiques.

24. Les 18 établissement d'enseignement supérieur et les 19 filiales d'établissements similaires de la Fédération de Russie, de même que les 38 écoles spécialisées et professionnelles, comptaient près de 20000 étudiants ; cf. GALSTYAN, 2004. 
russophone étaient d'un meilleur niveau que les lycéens formés à l'école azérie (Exo, 23 janvier 2003). Le maintien de la russophonie à Bakou ne doit pourtant pas cacher son repli à l'échelle du pays où le nombre d'écoles russophones est en baisse et le russe de moins en moins enseigné dans les écoles azerbaïdjanophones - citons, à titre d'exemple, le district de Kazakh où, en 2003, seuls 60 élèves sur 14000 étudiaient le russe (Ibid.).En Géorgie, sur près de 3100 écoles publiques, il restait, en 2005, 551 écoles nationales, dont 239 russophones ${ }^{25}$. Dans certaines d'entre elles, les minorités non russes (Arméniens, Azerbaïdjanais, Assyriens, etc.) constituaient la majorité des élèves, ce qui les dévalorisait aux yeux des Russes. Quoi qu'il en soit, la mixité ethnique, qui n'était pas rare dans ces écoles, tend à le devenir. En effet, même quand leur principe n'est pas remis en question, les écoles nationales sont paradoxalement la cible d'une ethnicisation des langues. Ainsi, en Géorgie, le ministre de l'Éducation du gouvernement de Zviad Gamsakhourdia (1990-1991) avait, dès son époque, souhaité encourager les langues nationales au détriment du russe et contraindre les enfants des minorités ethniques à faire leurs études dans leur langue éponyme ${ }^{26}$.

Dans les trois pays du Caucase, l'apprentissage de la langue nationale est devenu obligatoire, le niveau exigé et le volume horaire consacré augmentant au fil des années (article 5 de la loi azerbaïdjanaise sur la langue, article 2 de la loi sur la langue de l'Arménie). Les épreuves obligatoires de langue nationale lors des examens d'entrée à l'université, introduites en Azerbaïdjan et en Géorgie depuis quelques années, sont de plus en plus exigeantes. En Géorgie, le nouveau système de tests nationaux mis en place en 2005 permettait de choisir le géorgien comme langue étrangère. Cependant, dès 2006, tous les étudiants ont été soumis à la même épreuve, qu'ils aient fait leurs études secondaires dans une école russophone ou géorgianophone. Il en est résulté l'échec aux examens d'entrée de la quasitotalité des élèves issus des écoles nationales au point qu'aucun étudiant n'a pu s'inscrire au département de littérature slave de l'Université de Tbilissi, tous les candidats ayant été « collés » à l'épreuve de géorgien ${ }^{27}$.

$\mathrm{Au}$-delà de la question linguistique, les réformes successives du système éducatif entraînent une "nationalisation » des contenus en élaborant des programmes scolaires dont certains visent explicitement la consolidation nationale (ceux d'histoire et de littérature géorgiennes notamment). En

25. Il peut s'agir d'écoles entières ou, de plus en plus, de certaines classes seulement. Cf . КоRTH et al., 2005.

26. Entretien avec Elizbar Djavelidzé, ancien ministre de la Culture et ministre de l'Éducation sous Z. Gamsakhourdia (Paris, 1993).

27. En Kvémo Kartlie, une région comportant de fortes minorités ethniques, sur 1000 candidats potentiels, seuls 100 se sont présentés et seuls 12 ont été reçus. Il s'agissait d'élèves sortant de l'école géorgienne ou issus de familles mixtes. Entretien avec A. Bejenceva, présidente de l'association Jaroslavna qui, entre autres, se consacre à la défense de la culture et des communautés russes (Tbilissi, avril 2007).

Volume 39, Mars 2008 
l'absence d'harmonisation des programmes et des diplômes, il devient de plus en plus difficile de faire des études en Russie après le secondaire. De fait, dans les écoles géorgiennes et azerbaïdjanaises - y compris russophones -, l'enseignement diffère tant de celui prodigué en Russie qu'il est impossible pour les bacheliers de réussir les tests d'entrée dans les universités russes. Pour surmonter ces difficultés, l'on a ouvert des universités dont les programmes sont alignés sur les programmes russes et qui dépendent généralement du ministère de l'Éducation de la Fédération de Russie - par exemple l'Université russo-arménienne créée à Erevan en 1998 ou l'Université slave de Bakou - ainsi que des filiales d'instituts d'enseignement supérieur russes ${ }^{28}$. Le problème tient alors au fait que les diplômés de ces établissements ne peuvent voir leur diplôme validé sur place, ce qui les condamne d'emblée à faire le choix migratoire. Aussi les écoles nationales sont-elles devenues une pépinière de futurs émigrants. En parallèle, c'est l'impossibilité d'effectuer ses études en russe qui est souvent évoquée comme l'une des motivations du projet migratoire (Strel'cova, 2001).

Notons que le russe a une fonction particulière de langue de communication interethnique, c'est-à-dire de langue véhiculaire et non simplement «nationale ». Cela a renforcé l'ambiguité du rapport au russe qui est d'autant plus affaibli qu'il n'a pas la légitimité des « langues maternelles ». Au-delà, cette situation renvoie à une autre ambiguïté qui concerne les Russes eux-mêmes : ils ne se perçoivent ni ne sont perçus comme une minorité ethnique semblable aux autres.

\section{L'IMPOSSIBLE MOBILISATION ETHNIQUE}

L'ancien système permettait aux différents groupes de population de vivre, non pas ensemble, mais côte à côte. Quand il disparaît et, avec lui, l'illusion d'une intégration dans la grande famille soviétique, les populations russes se retrouvent marginalisées, étrangères dans leur pays. À l'instar des autres minorités, elles sont fragilisées par les discours ou pratiques racistes mais aussi par l'absence de représentation politique.

\subsection{Des Citoyens DE SECONDE ZONE}

En dépit de la reconnaissance formelle de l'égalité des citoyens quelle que soit leur origine, les Russes sont confrontés à de notables difficultés d'insertion liées à l'absence de réseaux familiaux, à la méconnaissance de la langue officielle mais aussi, et surtout, à la prédominance du prisme ethnique. En effet, en Géorgie, Arménie et Azerbaïdjan, c'est l'« ethnie » et non la nation dans son acception civique qui, pour l'ensemble des populations, constitue la catégorie structurante. Beaucoup, y compris parmi les

28. L'absence d'accord-cadre rend impossible l'ouverture de tels établissements en Géorgie. 
minorités, ont même la conviction que la prévalence de l'ethnie titulaire sur son territoire s'inscrit dans un ordre politique naturel dont il convient seulement de limiter les abus. Ce cadre idéologique partagé disqualifie tout discours d'adhésion civique de type contractuel, orienté vers un projet social et politique commun, dont l'échec peut être lié à celui de la " démocratisation ». Toutefois, en Azerbaïdjan et surtout en Géorgie, où les minorités constituaient $30 \%$ de la population en 1989, les modèles consociatif ou communautariste n'ont pas été érigés en alternative.

Les populations non titulaires ont été confrontées à des perceptions, des discours et parfois des pratiques discriminatoires. Celles-ci étaient particulièrement vigoureuses en Géorgie et en Azerbaïdjan où la présence massive de groupes ethniques minoritaires exacerbait la question nationale. Les Russes n'ont pas été épargnés. Ainsi, lors d'une rencontre publique entre le leader nationaliste Merab Kostava ${ }^{29}$ et les représentants des minorités à Abastoumani, au sud de la Géorgie, celui-ci a assuré que "pour le moment, les militants du mouvement national ne toucheraient pas aux Doukhobores ${ }^{30}$. Dans l'ensemble, l'attitude à l'égard des Russes n'est pas franchement hostile et les stéréotypes les concernant ne sont pas aussi marqués que pour d'autres minorités. En effet, en Géorgie, ils ne sont ni détestés comme les Arméniens ni méprisés comme les Kurdes, mais tout simplement oubliés. On ne trouve pas de déclarations publiques officielles explicitement russophobes comme cela peut être le cas pour d'autres groupes $^{31}$. L'image qui prévaut est celle de populations à plaindre, vues comme les victimes trop passives d'un État russe répressif et irrespectueux des individus. La distinction est donc très nettement faite entre Moscou et les Russes vivant sur place. Alors que la crise des relations russo-géorgiennes à l'automne 2006 a donné lieu à une véhémente campagne raciste anti-caucasienne en Russie, rien de tel n'a été observé en Géorgie où les populations russes n'ont pas été prises à partie et ne sont pas tenues pour comptables de la politique de la Fédération de Russie. Ces dernières font preuve d'une inertie politique relevant certes du stéréotype, mais aussi de l'injonction puisque leur dissociation de l'État russe est l'une des conditions de la mansuétude à leur égard.

Le signe le plus tangible de cette difficulté d'intégration est l'éviction des populations russes de la représentation politique. Les quotas impli-

29. Compagnon de route de Z. Gamsakhourdia, il meurt dans un accident en 1989.

30. Entretien avec A. Bejenceva, Tbilissi, avril 2007.

31. Pour ne donner qu'un exemple : en Géorgie, un député de la majorité justifiait récemment un projet de loi prévoyant de taxer lourdement les habitants des quartiers historiques de Tbilissi. Il précisait que cette loi ne visait pas les Géorgiens : « dans ces quartiers historiques vivent des Arméniens, des Azerbaïdjanais, des Kurdes et d'autres nationalités » (Radio Ucnobi, Dialogue). Toujours selon lui, «il n'est pas indispensable que Mkrtum [un prénom typiquement arménien] vive perpétuellement à Sololaki [un quartier central du vieux Tbilissi] » (TV Imedi), 24 saati, 12 avril 2007.

Volume 39, Mars 2008 
cites appliqués aux différentes nationalités sous le régime soviétique ont peu à peu disparu, si bien qu'en 2007, il n'y a plus aucun député russe en Géorgie ni en Arménie. En Azerbaïdjan, le seul élu est le président de la communauté russe, désigné au scrutin de liste en tant que membre du Parti « Nouvel Azerbaïdjan » (le parti officiel). Les Russes ne vivant pas en peuplement compact, ils ne peuvent comme d'autres groupes tels les Grecs ou les Arméniens bénéficier de réseaux, même à l'échelle locale, ni espérer être représentés par les députés de leur circonscription. La situation n'est pas plus brillante dans les instances exécutives: aucun des trois pays ne compte de ministre ou vice-ministre qui soit russe. En conséquence, même si les droits civils des Russes sont formellement reconnus, ils ne sont pas pleinement exercés.

\subsection{L'IMPOSSIBLE ABANDON DE L'IDENTITÉ IMPÉRIALE}

Les populations russes manquent des ressources politiques et symboliques sur lesquelles s'appuyer pour inventer leur mode d'intégration dans les États caucasiens. Leur difficulté à s'intégrer en tant que citoyens se double de leur incapacité à mobiliser le cadre ethnique et cela, pour deux raisons : la première tient à la fluidité des frontières du groupe, la seconde à une vision supra-ethnique portée par la réminiscence d'un discours «impérialiste » mettant l'accent sur l'action civilisatrice des Russes au Caucase.

\subsubsection{Le flou identitaire}

E. Poppe et L. Hagendoorn (2001) font remarquer que le terme de «Russes du Caucase » ne fait pas grand sens dans la mesure où il ne s'agit pas d'une communauté consciente d'elle-même, mais d'une pluralité d'individus ou de groupes qui obéissent à des logiques sociales propres et ne partagent ni le même mode de vie ni la même histoire ni le même rapport au pays dans lequel ils vivent. L'identité présumée des Russes du Caucase est donc particulièrement problématique à divers titres. D'une part, la disparité du groupe ne permet guère de réunir autour d'éléments communs des populations aussi hétérogènes que celles qui sont urbaines et travaillent dans l'industrie ou font partie de la petite intelligentsia technique et d'autres qui, rurales, ont longtemps été majoritaires en Arménie et sont massivement présentes en Azerbaïdjan; elles-mêmes sont d'ailleurs très hétérogènes et vivent selon leurs propres règles, sans beaucoup d'interactions avec l'extérieur. D'autre part, la différenciation des populations qui appartiennent à une aire culturelle russe dont les déterminants sont vagues est peu prononcée et récente. Les institutions soviétiques ont certes joué un rôle dans l'assignation de cette identité mais celle-ci est longtemps restée relativement peu investie, faute d'enjeux jugés fondamentaux. 
Aussi convient-il de parler d'une affinité culturelle reposant sur des facteurs multiples plutôt que d'une identité nationale. La notion de "slavité » est importante, du moins dans un premier temps. Ainsi, au début des années 1990, en Géorgie, s'était constitué un mouvement dit « la maison slave » qui regroupait, entre autres, des Russes, des Ukrainiens, des Biélorusses, des Bulgares et des Polonais. Aujourd'hui, au contraire, Russes et Ukrainiens tendent à être différenciés, les derniers étant choyés par les autorités géorgiennes du fait des bonnes relations bilatérales entre les deux pays depuis les révolutions « des Roses » et « orange $»^{32}$. La religion est également un facteur de poids dans la construction de ces identités qui justifie, par exemple, une proximité entre les Géorgiens et les Grecs. Les Allemands russifiés, les Juifs ashkénazes (appelés sur place « Juifs russes », à la différence des Juifs caucasiens, géorgiens ou des montagnes) sont considérés comme des Russes en raison du facteur linguistique ; en Arménie, les sectateurs ont longtemps été totalement assimilés aux Russes.

\subsubsection{Russes, Russiens, Soviétiques ${ }^{33}$ ?}

La question identitaire des Russes du Caucase se rapporte aussi à la difficulté de définir la «russité » dans l'ensemble de l'espace postsoviétique et à la perpétuation d'une identité soviétique parmi les Russes de l'«étranger proche » (Poppe \& Hagendoorn, 2001). A. Regamey montre à partir de la culture populaire que l'ambiguïté a toujours été de mise et que, dans le domaine symbolique, les Russes étaient «plus égaux » que d'autres ${ }^{34}$. Comment se penser en tant qu'ethnie alors qu'on est un empire ? Et, plus complexe encore, comment se penser comme une «minorité » dans un petit pays périphérique alors que l'on représente un peuple de 120 millions d'habitants?

L'incertitude identitaire est d'autant plus douloureusement ressentie que le passé commun est déformé et rejeté dans les discours publics officiels tandis que la culture russe, considérée comme intrinsèquement impériale, est décriée. Depuis l'indépendance, les marques du passé liées à la Russie s'effacent de l'espace public (nationalisation des toponymes, retrait des monuments dédiés non seulement aux héros soviétiques mais encore aux écrivains russes, à l'instar de la statue de Gogol déboulonnée au début des années 1990 à Tbilissi). Quelques jours après le dépôt des cendres de Z. Gamsakhourdia au panthéon géorgien, le député et proche conseiller

32. Le monument érigé à Chevtchenko, l'ouverture d'écoles ukrainiennes, etc. sont des signes tangibles de ce rapprochement. Sur l'instrumentalisation des minorités nationales dans la politique extérieure géorgienne, voir SERRANO, 2007.

33. La langue russe fait la distinction entre les Russes « ethniques » (russkie) et l'ensemble des citoyens de la Fédération de Russie, qui peuvent appartenir à d'autres groupes ethniques (rossijane).

34. Amandine Regamey (2007) cite ainsi une častuška, sorte de refrain populaire : « Comme c'est bien que Gagarine / Ne soit ni juif ni tatar / Pas un de ces culs-noirs / Mais un vrai Soviétique ».

Volume 39, Mars 2008 
du président, G. Bokéria, réclame le retrait de celles de Griboïedov qui, quoique "poète de génie », n'en était pas moins un "célèbre diplomate au service de son pays, l'Empire russe », et non un Géorgien ${ }^{35}$. Les populations russes du Caucase s'émeuvent de ce révisionnisme qui les renforce dans leur nostalgie de la mission civilisatrice de leurs ancêtres. Un journaliste russe pouvait ainsi commenter : « Griboïedov a joué un rôle important à Tbilissi, Bokéria, lui, qu'a-t-il fait ? $»^{36}$. Plus incompréhensibles encore, dans la patrie de Staline et de Béria, sont le regard porté sur la période soviétique et les tentatives de faire endosser à la Russie l'entière responsabilité des crimes et des échecs de l'URSS. Les revendications en faveur de la «lustration » tournent $\operatorname{court}^{37}$ tandis qu'un «musée de l'occupation soviétique » ouvre finalement ses portes au sein du Musée historique.

Le tâtonnement terminologique qui permettrait de définir un statut acceptable n'a pas retenu l'expression «minorités nationales» car elle renvoie trop clairement aux colonisés et n'est pas envisageable pour les Russes. Les deux termes qui s'imposent sont ceux de « diaspora » et de «compatriotes». Le premier permet de reconsidérer les liens avec la Russie tout en faisant le deuil de la fusion antérieure. Certains auteurs en récusent l'usage au prétexte qu'il supposerait des liens forts avec le pays d'origine (Poppe \& Hagendoorn, 2001). Néanmoins, se concevoir comme une diaspora suppose un enracinement sur place ou, pour le moins, ne fait pas de l'émigration le seul horizon possible.

Le second terme, sootečestvennik (compatriote), illustre bien l'ambiguïté de l'identité des «pieds rouges ». Il est utilisé dans la loi de la Fédération de Russie sur « les compatriotes vivant à l'étranger $»^{38}$, ainsi que dans le décret présidentiel qui prévoit un nouveau programme d'aide au retour ${ }^{39}$.

35. Voir par exemple, Kommersant, 13 avril 2007.

36. Aleksandr Svatikov, rédacteur en chef de la revue Russkij Klub, Tbilissi, avril 2007. La revue est consultable sur le site : http://www.russianclub.ge

37. On entend par lustration une opération de «purification et de réconciliation » qui a touché les administrations des pays d'Europe centrale et orientale après 1989. Ce processus a été notamment marqué par la publication de listes de collaborateurs des services secrets des anciens régimes communistes. En Géorgie, lors des discussions sur l'opportunité d'une telle opération, certaines personnalités se prononcent en faveur de poursuites non seulement contre les personnes soupçonnées d'avoir collaboré avec le KGB durant la période soviétique, mais aussi contre celles qui ont des liens avec des organisations russes actuelles. Ainsi, l'ONG Liberty Institute juge bon que tous ceux qui ont été en contact avec Modest Kolerov (président du Département pour les liens interrégionaux et culturels avec les pays étrangers de la Fédération de Russie) en réfèrent immédiatement aux autorités géorgiennes. Voir Civil Georgia, 21 février 2007.

38. Loi fédérale n ${ }^{\circ}$ 99-F3 du 24 mai 1999 « Sur la politique de la Fédération de Russie dans ses relations avec les compatriotes de l'étranger » (Federal'nyj zakon o gosurdarstvennoj politike Rossijskoj Federacii v otnošenii sootečestvennikov za rubežom), Sobranie zakonodatel'stva Rossijskoj Federacii (SzRF), 1999, n 22, art. 2670.

39. Décret présidentiel $n^{\circ} 637$ du 22 juin 2006 « Sur les mesures d'aide au rapatriement volontaire en Fédération de Russie des compatriotes vivant à l'étranger » ( $\mathrm{O}$ merakh po oka- 
Les fonctionnaires chargés de les appliquer bénéficient d'une marge d'appréciation importante car ces textes ne délimitent pas clairement les catégories de population concernées. La loi définit comme compatriotes « ceux qui ont la citoyenneté russe et vivent à l'étranger (les ressortissants de l'exURSS) et ceux qui vivent actuellement dans les États de l'ex-URSS (qu'ils aient ou non acquis la citoyenneté de leur pays de résidence), ainsi que les émigrés qui ont eu la citoyenneté russe, même s'ils ont depuis acquis une autre citoyenneté » (Rapport de la FIDH, 2007). Le décret de 2006 les qualifie de personnes «éduquées dans les traditions de la culture russe, maîtrisant le russe et ne souhaitant pas perdre leurs liens avec la Russie » (Ibid.). En théorie, les programmes concernent donc l'ensemble des populations nées en URSS mais, implicitement, ciblent les populations russophones, les « russophones sans accent $»^{40}$. Compte tenu de ce flou, un grand nombre d'Arméniens cherchent à en bénéficier, alors que les diplomates russes s'interrogent sur la recevabilité de telles demandes ${ }^{41}$.

\subsubsection{La faiblesse des mobilisations identitaires}

L'incertitude identitaire liée à leur statut passé dans l'empire soviétique prive les Russes de l'atout majeur - la possibilité d'une mobilisation ethnique - qu'ont eu en main les autres minorités, du moins en Géorgie et en Azerbaïdjan ${ }^{42}$. Dans ces conditions, les organisations communautaires font en général profil bas et s'impliquent peu dans le débat public. Ainsi, lors des discussions sur la validité des différents modèles d'intégration civique en Géorgie (Serrano, 2008), les Russes n'ont pas été partie prenante, leurs capacités de peser étant réduites et leur légitimité à participer au débat public moindre que celles des populations autochtones.

En outre, ils souffrent de l'absence de certaines des institutions - les Églises notamment - qui, dans le cas d'autres groupes, jouent un rôle important. Les églises orthodoxes russes sont en effet sous la juridiction de l'Église autocéphale géorgienne et, de fait, la messe n'est plus dite en russe que dans une seule église à Tbilissi. En Azerbaïdjan, l'éparchie de la Caspienne est trop faible pour constituer un pôle de ralliement ${ }^{43}$. Les

zaniju sodejstvija dobrovolnomu pereselenija v Rossijskuju Federaciju sootečestvennikov proživajuščikh za rubežom), $S z R F, 2006, \mathrm{n}^{\circ} 26$.

40. Selon le terme utilisé par un journaliste de NTV (http://news.ntv.ru/itogi/100018/). Cité par le rapport de la FIDH (2007) qui souligne le fait que, davantage « que les questions juridiques, ce sont la capacité d'assimilation, la maîtrise de la langue et la familiarité avec la culture russe (des qualités que possèdent les personnes ayant grandi en URSS, avec son système scolaire centralisé et unifié, ses médias uniques, etc.) qui semblent importer ».

41. Entretiens avec Valerij Anatolovič Vasilev, consul, chargé du programme de rapatriement des Doukhobores, Tbilissi, avril 2007.

42. En Arménie, la question se pose en d'autres termes du fait de la faiblesse numérique des Russes et, partant, de la quasi-inexistence d'organisations collectives.

43. Restaurée en septembre 1998 par le synode de l'Église orthodoxe russe, l'éparchie de Bakou-Kaspiïskaïa (Azerbaïdjan, Daghestan, Tchétchénie) comprend six paroisses en 
différentes organisations communautaires ${ }^{44}$, peu influentes, s'y distinguent difficilement des organes officiels avec lesquels elles collaborent. La proximité est parfois manifeste, ce dont témoigne le «Conseil républicain des communautés russes » qui a un bureau dans les bâtiments du gouvernement ${ }^{45}$. En Géorgie, elles revendiquent principalement une mission culturelle ou se consacrent aux problèmes quotidiens spécifiques à certaines petites communautés comme les Doukhobores ${ }^{46}$. À l'instar de toutes les associations en Géorgie, elles se substituent parfois aux services publics défaillants, par exemple en organisant des formations pour adultes de langue géorgienne ou d'informatique. Toutefois, les projets ciblant les Russes peinent à attirer les financements et leurs responsables avouent parfois «gommer» leur spécificité nationale pour leur donner la forme de programmes «multiethniques », plus aisément vendables ${ }^{47}$.

Les effets du déclin des institutions, qu'elles soient formelles ou informelles, dépassent les limites des frontières nationales. Depuis la « révolution des Roses » géorgienne, les relations entre les associations communautaires et les autorités russes pâtissent de l'absence de ces institutions qui servaient de courroie de transmission entre les autorités et les populations russes ; sans elles, il devient ardu de trouver les interlocuteurs adéquats ${ }^{48}$. D'autres organisations ou personnalités jouent les bons offices auprès des autorités russes ou de groupes de pression en Fédération de Russie ${ }^{49}$. Il convient de souligner que, paradoxalement, de tels soutiens officiels ne favorisent pas toujours les associations locales : ils limitent leur capacité d'intervention dans les pays où elles se trouvent car elles sont soupçonnées d'être la cinquième colonne.

Les organisations qui pourraient exprimer les revendications des populations russophones et défendre leurs intérêts sont donc doublement entravées, d'un côté par la faiblesse de l'identification qui leur interdit de jouer de la mobilisation ethnique, de l'autre par les effets pervers d'un soutien

Azerbaïdjan, dont trois à Bakou, une à Gandja, une à Soumgaït et une dans le district de Khatchmaz. Elle est fréquentée par des Russes, mais aussi par des Ukrainiens, des Biélorusses, des Grecs et des Moldaves. Voir Galstyan, 2004.

44. La Communauté russe de la République d'Azerbaïdjan (la plus importante du pays avec 28 représentations dans les régions et plus de 70000 membres enregistrés), le Centre culturel slave, le Centre culturel azerbaïdjano-slave, la société Sodružestvo, l'Amicale des Cosaques ; voir GALSTYAN, 2004.

45. Lire l'interview avec le président de l'association; http://www.azeri.ru/AZ/etno/zabelin.html 46. Telles les associations « Russkij klub », «Etno », « Jaroslavna ».

47. A. Bejenceva a appelé son ONG «Géorgie multethnique » après avoir constaté qu'un même projet obtenait plus facilement des financements s'il visait les minorités ethniques en général que s'il était exclusivement destiné aux Russes.

48. Le responsable des minorités nationales à la Chancellerie sous la présidence d'E. Chevardnadzé, A. Guérassimov, n'a pas été remplacé et la ministre d'État à l'Intégration, Zinaïda Bistaeva, est peu active.

49. Par exemple, l'Union des compatriotes russes de Géorgie qui regroupe 27 associations. 
potentiel de l'ancienne métropole. Ce dernier est néanmoins loin d'être manifeste. En effet, plus que d'une politique de puissance, les Russes du Caucase sont victimes de la faiblesse de la Russie.

\subsection{UNE CARTE QU'ON NE JOUE PAS}

Dans le Caucase, certaines minorités (les Arméniens, les Azéris, les Grecs) jouissent d'une ressource non négligeable : la présence d'un État titulaire susceptible d'intervenir diplomatiquement pour défendre leurs intérêts. Celle-ci manque aux populations russes tant la Fédération de Russie s'est désintéressée d'elles.

\subsubsection{Une politique d'influence embryonnaire}

Dans leur ensemble, les militants associatifs ou culturels russes déplorent l'insuffisance de l'aide émanant de la Fédération de Russie, voire son absence comme le rapporte le directeur du théâtre Griboïedov, le seul théâtre russophone aujourd'hui en Géorgie, qui affirme ne recevoir aucune aide officielle russe, si ce n'est quelques billets achetés par l'ambassade pour son personnel ${ }^{50}$. Le budget annuel total alloué aux programmes éducatifs et culturels russes en Géorgie est de 312000 dollars depuis $2005^{51}$, une grande part allant à diverses rencontres de 4 compatriotes $»^{52}$. Les subventions russes destinées à des programmes de coopération culturelle sont minimes et aucun argent public n'est versé aux « Journées de la culture russe ». De même, et c'est significatif, aucune ligne budgétaire n'a été ouverte en Géorgie dans le cadre de l'année de la langue russe lancée par le président Poutine en 2007.

L'aide de la Fédération de Russie dans le domaine éducatif est, elle aussi, très limitée. En Arménie, la Russie prend en charge la formation des enseignants de russe, fait don de manuels aux écoles russophones ou a contribué à la restauration de l'école russe de Gumri (Nesavisimaja gazeta, 26 mai 2001). Elle finance également les études, dans la Fédération russe, des boursier retenus sur concours ${ }^{53}$. En Géorgie, les associations regrettent l'absence de centre culturel russe équivalent au British Council ou au Goethe Institut, alors que les cours de russe sont dispensés... par le centre culturel français (le Centre Dumas) ${ }^{54}$.

50. Entretien avec Nikolaj Svensickij, directeur du théâtre Griboïedov, Tbilissi, avril 2007. Notons que le public de ce théâtre est majoritairement géorgien. Il existe également un théâtre russe à Bakou.

51. Entretien avec Vasilij S. Korčmar, conseiller de l'ambassade de la Fédération de Russie en Géorgie, avril 2007.

52. Congrès des compatriotes, séminaires de formation, etc.

53. Soixante-dix étudiants par an sont concernés en Géorgie.

54. L'ouverture, en mars 2007, d'une « Maison russe » à Erevan où ne vit pratiquement aucun Russe suscite l'incompréhension. 
Les seules initiatives concrètes ont concerné le rapatriement (pereselenie) des Doukhobores ${ }^{55}$. Une première vague de retour avait été organisée en 1998 vers la Russie centrale (région de Briansk). En 2007, quelque 200 personnes devaient quitter les villages du district de Ninotsminda et s'installer, pour la plupart, dans la région de Tambov. On peut s'interroger sur la pertinence de ce programme dans la mesure où les populations visées, pour dures que soient leurs conditions de vie, ont moins souffert du déclassement que celles des centres industriels. Il semble par ailleurs que leur retour en Russie se soit accompagné d'un abandon total de leur mode de vie traditionnel et de leurs pratiques religieuses, les jeunes cherchant à rejoindre les grandes villes. Il est donc difficile de ne pas voir là un projet consensuel qui puisse satisfaire les autorités géorgiennes et russes, ainsi que les populations arméniennes locales qui s'opposent aux Doukhobores depuis le début des années $1990^{56}$.

Le soutien politique aux populations russes est également très restreint. Ainsi, officiellement, il n'est pas plus facile pour les Russes dotés d'un passeport géorgien d'obtenir un visa pour la Russie que pour les éponymes. On comprend donc que les représentants des communautés russes sont généralement fort dépités face à une Russie «qui les a abandonnés à leur triste sort $\aleph^{57}$. Paradoxalement, les rares mobilisations des populations russophones ont été dirigées contre la politique de Moscou. Quand, à l'automne 2006, la décision a été prise de fermer l'école russe qui dépendait des Forces russes en Transcaucasie - une mesure manifeste de rétorsion en pleine «crise des espions »-, des rassemblements de protestation ont eu lieu. La totalité des 300 parents d'élèves - russes ou non - a signé une lettre ouverte adressée à l'épouse de Vladimir Poutine, lui demandant « de conserver l'école en tant que centre de la langue et de la culture russes » et présentant les élèves, « attirés par la culture russe et l'enseignement russe, comme des passeurs œuvrant, en dépit des tensions et de la complexité des rapports entre les deux pays, aux relations de bon voisinage $»^{58}$.

Ce bilan est probablement à nuancer par la prise en compte des fonds privés qui esquissent de nouvelles politiques d'influence. Les représentants des institutions culturelles russes reconnaissent qu'une part de leurs finan-


russes de Tbilissi ou de Bakou.

56. Au début des années 1990, pour renforcer le poids démographique des Géorgiens dans une région peuplée d'Arméniens à plus de $90 \%$, le Fonds Kostava, du nom d'un ancien dissident et leader du mouvement national, a racheté les maisons des Doukhobores de Bogdanovka (aujourd'hui Ninotsminda) pour 5 000-10 000 dollars US, soit le prix d'un appartement dans le centre de Tbilissi. Depuis plusieurs années, les Arméniens cherchent à s'installer dans ces villages et poussent les Doukhobores au départ, quand ils ne les exploitent pas à leur profit.

57. Entretien avec Nikolaj Svensickij, Tbilissi, avril 2007.

58. Voir à ce sujet, Komsomol'skaja pravda v Gruzii, 24-30 novembre 2006 ; http://www.pankisi.info/media/?page $=$ ge \&id $=9405$ 
cements provient de mécènes dont la plupart, semble-t-il, sont de riches hommes d'affaires caucasiens installés en Russie. La frontière entre fonds privés et politiques publiques est toutefois brouillée. Ainsi, l'une des sources de financement des activités culturelles russes en Géorgie serait une fondation créée par Modest Kolerov; elle est présentée comme une institution privée alors que celui-ci est président du Département pour les liens interrégionaux et culturels avec les pays étrangers et, à ce titre, directement sous l'autorité du chef de l'Administration présidentielle ${ }^{59}$. S'il fallait y voir une tentative de Moscou d'instrumentaliser la politique culturelle dans le cadre de sa politique étrangère, le moins qu'on puisse dire est que ses effets sont pour l'instant fort modestes.

\subsubsection{Un enjeu de faible intensité}

Lors de sa visite officielle en Arménie en mars 2005, le président Vladimir Poutine n'avait pas prévu de rencontrer les représentants de la communauté russe locale, signe à ses yeux du peu d'intérêt que Moscou lui prêtait (Galstyan, 2004). En effet, les Russes du Caucase méridional ne constituent pas une carte que Moscou entend jouer et représentent manifestement un enjeu de très faible intensité. Trop peu nombreux, ils ne sauraient être l'instrument d'une reconquête ; ils ne sont même pas les otages des tensions bilatérales et, selon toute apparence, sont purement et simplement ignorés. La principale raison tient à leur faiblesse démographique, la situation n'étant en rien comparable avec celle qui prévaut en Ukraine ou au Kazakhstan.

La passivité à l'égard des Russes de Géorgie tranche avec la politique très offensive menée par Moscou envers les Abkhazes et les Ossètes, notamment par le biais d'une campagne d'octroi de passeports russes qui pourrait servir aux autorités à justifier une intervention dans ces régions afin de protéger des concitoyens. Le Kremlin préfère donc jouer la carte des minorités ethniques plutôt que celle des Russes et a soutenu, même au-delà des régions séparatistes et plus ou moins ouvertement, les revendications de certains groupes tels les Arméniens de Djavakhétie, région dans laquelle était implantée l'une des quatre bases militaires russes de Géorgie, aujourd'hui en cours d'évacuation.

Lorsque la Fédération de Russie a cherché à « intervenir » dans les affaires intérieures, c'est toujours en tentant de promouvoir des Azerbaïdjanais ou des Géorgiens de souche. Il faut probablement y voir une continuité avec les pratiques impériales de cooptation des élites, ainsi qu'un souci d'efficacité, seule une personnalité autochtone pouvant jouir d'une légitimité suffisante ${ }^{60}$. Dans ses timides tentatives de conversion au soft power,

59. Pour plus de détails, lire Kommersant, 23 mars 2005 (http://www.kommersant.com/page. asp?id=556859. Ce poste avait été créé dans la foulée de la « Révolution orange » en Ukraine.. 60. C'est la politique qui a été menée en Tchétchénie. Il faut cependant en noter les limites 
elle a également pu financer des ONG ou des fondations sur le modèle occidental en prenant soin de nommer à leur tête des membres de la nation titulaire et non des Russes ${ }^{61}$.

Les Russes du Caucase sont ainsi victimes de la faiblesse des enjeux liés à leur présence dans cette région. Les autorités russes se soucient peu de soutenir leurs compatriotes lorsque les gains politiques qu'elles pourraient en tirer sont négligeables. Cette approche encore explicitement utilitariste du soft power témoigne de la difficulté de la Russie à se projeter dans une vision stratégique et révèle sa volonté de privilégier les tactiques réactives à court terme. Le contraste avec les pays baltes est frappant : le poids démographique des Russes et, surtout, leur situation géographique incitent le gouvernement à les instrumentaliser dans ses relations avec l'Union européenne. Quand aucun bénéfice immédiat n'est à attendre - et comment le serait-il avec des populations appauvries et vieillissantes ? -, le pouvoir se désintéresse de ses compatriotes.

\section{ANNEXES}

Azerbaïjjan

\begin{tabular}{|l|c|c|c|c|c|}
\hline & 1939 & 1959 & 1979 & 1989 & 2001 \\
\hline $\begin{array}{l}\text { Nombre de } \\
\text { Russes }\end{array}$ & 528000 & 501300 & 475300 & 392300 & 141700 \\
\hline $\begin{array}{l}\text { Part de la } \\
\text { population }\end{array}$ & $16,5 \%$ & $13,6 \%$ & $7,9 \%$ & $5,6 \%$ & $1,8 \%$ \\
\hline
\end{tabular}

Source : SKAKOV, 2003.

Arménie

\begin{tabular}{|l|c|c|c|c|c|}
\hline & 1939 & 1959 & 1979 & 1989 & 2001 \\
\hline $\begin{array}{l}\text { Nombre de } \\
\text { Russes }\end{array}$ & & 56700 & 70400 & 51600 & 14660 \\
\hline $\begin{array}{l}\text { Part de la } \\
\text { population }\end{array}$ & $3,2 \%$ & $2,3 \%$ & $1,6 \%$ & $0,45 \%$ \\
\hline
\end{tabular}

Sources : ANDERSON \& SiLvER, 1996 et le recensement de 2001 (http://docs.armstat.am/census/engcontent.php).

au Caucase méridional. En Azerbaïdjan, elle a pu aboutir au renversement d'A. Eltchibey en 1993, mais elle a été un échec en Géorgie où les «hommes de Moscou » ont été évincés et totalement décrédibilisés.

61. C'est le cas, par exemple, de la Fondation Igor Guiorgadzé dont la présidente, Irina Sarichvili, fut l'une de figure de proue du mouvement national ; c'est aussi celui de l'Institut caucasien pour la paix et la démocratie qui possède une filiale dans chacune des capitales du Caucase méridional et dont la présidente géorgienne, également membre du mouvement Anti-Soros, Maïa Nikolaïchvili, est aujourd'hui en prison depuis l'offensive des autorités contre les organisations accusées de préparer un coup d'État à l'automne 2006. 
Géorgie

\begin{tabular}{|l|c|c|c|c|c|}
\hline & 1939 & 1959 & 1979 & 1989 & 2002 \\
\hline $\begin{array}{l}\text { Nombre de } \\
\text { Russes }\end{array}$ & 407900 & 372000 & 341200 & 67671 \\
\hline $\begin{array}{l}\text { Part de la } \\
\text { population }\end{array}$ & $10,1 \%$ & $7,2 \%$ & $6,3 \%$ & $1,5 \%$ \\
\hline
\end{tabular}

Sources : Anderson \& SiLVER, 1996 et le recensement de 2002 (Sakartvelos mosaxleobis, 2002).

\section{RÉFÉRENCES BIBLIOGRAPHIQUES}

Anderson Barbara A. \& Silver Brian B. (1996), « Population Redistribution and Ethnic Balance in Transcaucasia », in Ronald G. Suny, Transcaucasia, Nationalism, and Social Change, Essays in the History of Armenia, Azerbaijan, and Georgia, Ann Arbor, Michigan: University of Michigan Press, pp. 481506 ( 1 ère édition en 1983).

Galstyan Garik (2004), « Les minorités russes dans le Sud-Caucase. Une diaspora en voix d'extinction », numéro spécial : Le Sud-Caucase, recomposition régionale et nouvelles alliances, Le courrier des pays de l'Est, $\mathrm{n}^{\circ} 1043$, mai-juin, pp. 24-36.

Jones Stephen (1992), "Georgia: The Long Battle for Independence », in M. Rezun, ed., Nationalism and the Breakup of an Empire: Russia and its Periphery, Westport: Praeger Press, pp. 73-96.

Kolstoe Paul (1995), Russians in the Former Soviet Republics, London: Hurst \& Co.

Korth Britta, Stepanian Arnold \& Muskhelishvili Marina (2005), Language Policy in Georgia with a Focus on the Education System, Rapport de la CIMERA, avril.

LAITIN David D. (1998), Identity in Formation, The Russian-Speaking Populations in the Near Abroad, Ithaca; London: Cornell University Press.

Layton Susan (1994), Russian Literature and Empire. The Conquest of the Caucasus from Pushkin to Tolstoy, Cambridge: Cambridge University Press.

Mouradian Claire (1990), L'Arménie, de Staline à Gorbatchev, histoire d'une république soviétique, Paris : Ramsay.

Olcott Martha \& Semenov Il'ja (2001), Jazyk i etničeskij konflikt (La langue et le conflit ethnique), Moskva: Gendal'f.

Poppe Edwin \& Hagendoorn Louk (2001), «Types of identification among Russians in the "Near Abroad" ", Europe-Asia Studies, Vol. 53, n ${ }^{\circ}$, pp. 57-71. 
Rapport de la FIDH - Fédération Internationale des Droits de l'Homme (2007), Les migrants en Russie. Des populations fragilisées, premières victimes des crises politiques internes et externes, $\mathrm{n}^{\circ} 472$, avril.

Regamey Amandine (2007), Prolétaires de tous pays excusez-moi, Paris: Buchet Chastel.

Rhinelander Anthony L.H. (1972), The Incorporation of the Caucasus in the Russian Empire. The Case of Georgia, 1801-1854, $\mathrm{PhD}$, Columbia University.

Rhinelander Anthony L.H. (1990), Prince Michael Vorontsov, Viceroy to the Tsar, Montréal: McGill-Queen's University Press.

Sakartvelos mosaxleobis 2002 c'lis saq'oveltao agc'era (2003) (Recensement général de la population de Géorgie, 2002), Tbilissi ; http://www.statistics. ge/main.php?pform $=108 \&$ plang $=2$

Serrano Silvia (2007), Géorgie, sortie d'empire, Paris : Éditions du CNRS.

Serrano Silvia (2008), «Les minorités face à l'État national, une nouvelle place à négocier : le cas de la Géorgie », in Marlène Laruelle \& Catherine Servant, dir., D'une édification l'autre. Socialisme et nation dans l'espace (post-)communiste, Paris : Éditions Pétra, pp. 251-287.

Shlapentokh Vladimir, Sendich Munir \& Payin Emil, eds. (1994), The New Russian Diaspora: Russian Minorities in the Former Soviet Republics, Armonk, New York: M.E. Sharpe.

SkAKov A. Ju (2003), « Etničeskaja i migracionnaja situacija v Azerbajdžane » (La situation ethnique et migratoire en Azerbaïdjan), in A. Iskandarjan, Migracii na Kavkaze, materialy konferencii, Erevan: Kavkazskij institut SMI, pp. 93-113.

StRel'Cova Jana (2001), « Problemy russkogo jazyka i obrazovanija v rossijskikh diasporakh v novom zarubežje » (Les problèmes de la langue russe et de l'éducation dans les diasporas russes du nouvel étranger), in Martha Olcott \& Il'ja Semenov, Jazyk $i$ etničeskij konflikt (La langue et le conflit ethnique), Moskva: Gendal'f ; http://www.carnegie.ru/ru/pubs/books/volume/36583.htm

Suny Ronald G. (1989), The Making of the Georgian Nation, London: Taurus.

Teurtrie David (2004), «La langue russe. Un enjeu dans les relations entre la Russie et son étranger proche », Le courrier des pays de l'Est, $\mathrm{n}^{\circ} 1043$, mai-juin, pp. 67-75.

Tinguy Anne de (2004), La grande migration. La Russie et les Russes depuis l'ouverture du rideau de fer, Paris : Plon. 
